\section{P081 AN AUDIT OF THE MANAGEMENT AND AETIOLOGY OF PROCTITIS IN MEN WHO HAVE SEX WITH MEN (MSM)}

Dean Gardner*, Patrick French. Mortimer Market Centre, Central North West London NHS Foundation Trust, London, UK

\subsection{6/sextrans-2016-052718.135}

Background Proctitis remains an important presentation of MSM to sexual health clinics. It causes significant morbidity and facilitates the transmission of other infections including HIV.

Aims To audit the management and aetiology of proctitis in a UK sexual health clinic and determine the pattern of STIs presenting with rectal symptoms.

Methods 100 consecutive notes of MSM presenting with rectal symptoms were examined (December 2014 - March 2015). The local clinical management standard is proctoscopy and Gram stain for gonorrhoea; gonorrhoea culture; gonorrhoea/chlamydia NAAT; HSV1/HSV2/T.pallidum PCR; syphilis serology. Positive chlamydia NAATs are tested for LGV-associated serovars.

Results 88/100 had proctoscopy performed. The tests undertaken and test results are summarised in the table below.

\begin{tabular}{llll} 
Abstract P081 Table 1 & Proctitis in MSM & & \\
\hline Investigation & $\begin{array}{l}\text { No. } \\
\text { undertaken }\end{array}$ & No. positive & $\begin{array}{l}\% \\
\text { positive }\end{array}$ \\
\hline Gram stain for gonorrhoea & 63 & 7 & 11.1 \\
Culture for gonorrhoea & 69 & 13 & 18.8 \\
NAAT for gonorrhoea & 97 & 24 & 24.7 \\
NAAT for chlamydia - non-LGV & 97 & 11 & 11.3 \\
serovar & & & \\
NAAT for chlamydia - LGV serovar & 97 & 10 & 10.3 \\
HSV1 PCR & 66 & 4 & 6.2 \\
HSV2 PCR & 66 & 10 & 15.1 \\
T.pallidum PCR & 66 & 3 & 4.5 \\
Syphilis serology & 94 & 7 (active & 7.4 \\
& & infection) & \\
\hline
\end{tabular}

43 patients had all the recommended tests. 66 infections were diagnosed in 53 patients. 42 patients had one infection, 9 had two infections and 2 had three infections. 35 patients were diagnosed HIV positive before presentation, 64 patients tested HIV negative at presentation and one patient declined testing.

Discussion This audit confirms that the majority of MSM presenting with rectal symptoms had proctoscopy but there is room for improvement in practice as only a minority had all tests undertaken. STIs are a common cause of anal symptoms in MSM and this data strongly supports a low threshold for STI screening. Routine HSV testing in MSM with rectal symptoms is useful.

\section{P082 EVALUATION OF A NEW LGBTI SERVICE TO COMPLEMENT A BUSY INNER CITY GUM CLINIC}

Eleanor Hamlyn*, Grainne Cooney, Kulvinder Randhawa, Sue Wood, Tristan Barber. Chelsea and Westminster Hospital, London, UK

10.1136/sextrans-2016-052718.136

Background/introduction LGBTI individuals are at significantly increased risk of STI's and HIV, as well as sexual violence and discrimination. The need for specialist LGBTI services in level 3 GUM settings is increasingly recognised and also subscribes to BASHH equality and diversity standards. We established a new LGBTI specialist clinic and present here a service evaluation of its first 8 months.

Aim(s)/objectives To evaluate a new LGBTI service.

Methods Coding for all patients who accessed the service over an 8 month period was collated and used to garner basic information about diagnoses. A 4 week period was then chosen at random and individual patient notes were accessed to get more detailed information.

Results There were 526 attendances for 450 individual patients. The rates of STI's compared to our general clinics are tabled below.

\begin{tabular}{lcl} 
Abstract P082 Table 1 & LGBTI Diagnoses & \\
\hline & LGBTI clinic & General GUM \\
\hline Gonorrhoea & $14.0 \%$ & $3.8 \%$ \\
Chlamydia & $6.4 \%$ & $5.0 \%$ \\
NSU & $5.3 \%$ & $2.9 \%$ \\
Syphilis & $3.8 \%$ & $1.2 \%$ \\
Warts & $4.8 \%$ & $6.3 \%$ \\
HSV & $2.0 \%$ & $4.0 \%$ \\
Treated as contact & $20.0 \%$ & $6.9 \%$ \\
\hline
\end{tabular}

In the 4 week period there were 104 booked attendances. The age range was 19 - 75 (mean: 37.1). Of the 92 patients who attended 59\% had at least one diagnosis with 13\% having multiple diagnoses. $26 \%$ were HIV positive.

Discussion/conclusion The high STI and HIV rates in this group suggest they will benefit from a specialist service. This involves a reconfiguration of staff compared to general clinics to account for increased requirements for treatments, injections and counselling. An additional qualitative assessment demonstrated that the clinic was also extremely well received by patients.

\section{P083 PSYCHOLOGICAL IMPACT DUE TO GENITAL HERPES AMONG CENTRAL STD CLINIC ATTENDEES IN SRI- LANKA}

${ }^{1}$ Nimali Jayasuriya*, ${ }^{2}$ Sivagurunathan Sivayogan, ${ }^{3}$ Kulasiri Buddhakorale. 'Guy's and St Thomas' NHS Foundation Trust, London, UK; ${ }^{2}$ University of Sri Jayawardenapura, Colombo, Sri Lanka; ${ }^{3}$ Dr. Neville Fernando Sri Lanka Russia teaching Hospital, Colombo, Sri Lanka

\subsection{6/sextrans-2016-052718.137}

Background/introduction Genital herpes is becoming the most prevalent STI throughout the world. Patients with genital herpes are more susceptible to psychological distress, possibly due to its natural history of incurability, asymptomatic viral shedding, recurrences, painful ulcers and risk of transmission to the partner and to the baby.

Aim(s)/objectives To study the psycho-social impact among patients with genital herpes.

Methods Study design was cross sectional comparative study using HSV infected and non-infected group attending central STD clinic Colombo. Study group was having genital herpes and a comparative group was asymptomatic and did not have genital herpes but having any other STI. Interviewer administered questionnaire was used for 85 from each group. General Health 